\title{
Intrauterine Growth Restriction: Guidelines for the use of Obstetrical Ultrasound
}

\author{
Giampaolo Mandruzzato
}

\begin{abstract}
Intrauterine growth restriction (IUGR) is a major problem in perinatal medicine. It is the second cause of perinatal mortality and morbidity after prematurity and the two conditions are frequently associated. The principal cause of the poor perinatal outcome is represented by the chronic fetal hypoxemia $(\mathrm{FCH})$ that occurs in 30 to $35 \%$ of IUGR fetuses. In order to improve significantly the clinical outcome a timely recognition and a proper management is fundamental. Today the method of choice for monitoring the fetal growth and detect any deviation is represented by serial fetal ulrtasound biometry. After suspicion or recogntion of fetal growth restriction it is necessary to assess the characteristics of the maternal-fetal exchanges in order to detedct or exlude the presence of chronic fetal hypoxemia.
\end{abstract}

Keywords: Chronic fetal hypexemia, Intrauterine growth restriction, Ultrasound fetal biometry and obstetrical Doppler.

How to cite this article: Mandruzzato G. Intrauterine Growth Restriction: Guidelines for the use of Obstetrical Ultrasound. Donald School J Ultrasound Obstet Gynecol 2016;10(3):350-351.

Source of support: Nil

Conflict of interest: None

\section{INTRODUCTION}

Intrauterine growth restriction (IUGR) is a major problem in perinatal medicine, being the second cause of perinatal mortality. The prevalence of this condition is 8 to $10 \%$ of all pregnancies.

The etiology can be maternal, fetal, or placental. The most frequent cause is placental obliterative vasculopathy. In this condition, nutrients and oxygen supply to the fetus are reduced, inducing growth restriction first and second chronic fetal hypoxemia (CFH) that affects 30 to $35 \%$ of IUGR fetuses.

In order to improve the outcome, a timely recognition and a rational management of IUGR is crucial.

Today ultrasound fetal biometry offers the best possibility for monitoring the fetal growth and obstetrical Doppler plays an important role in the management of $\mathrm{CFH}$.

Former Chief

Department of Obstetrics and Gynecology, IRCCS Burlo Garofolo, Trieste, Italy

Corresponding Author: Giampaolo Mandruzzato, Former Chief, Department of Obstetrics and Gynecology, IRCCS Burlo Garofolo, Trieste, Italy, e-mail: mandruzzatogiampaolo@tin.it

\section{FETAL GROWTH MONITORING AND IUGR RECOGNITION}

In order to recognize precisely any clinical condition, its precise definition is fundamental. In the case of IUGR, this is a very critical point as also recently more than 30 different definitions have been proposed. ${ }^{1}$

Many of them are based on estimated fetal weight (EFW) inferior to the 10th percentile for the gestational age (GA). This approach is an important source of errors for two reasons.

First, by using any proposed formula for EFW, the error, in defect or in excess, is not less than $10 \%$ of the actual weight. The consequence is at least $10 \%$ rate of false positives and negatives can be expected.

Second, but not less important, is the fact that in this way all the fetuses showing an EFW superior to the 10th percentile, but have suffered a restriction of their potential growth, will be missed.

Actually, it has been shown that $\mathrm{CFH}$, suggesting growth restriction, can be found in about $7 \%$ of fetuses presenting a birthweight (BW) between the 10th and the 25th percentile therefore considered as appropriate for GA but having suffered of failure to reach their potential growth. ${ }^{2}$

It has been proposed to define as IUGR fetuses after observation of restriction of ultrasound growth parameters from the projected curve by serial measurements. ${ }^{3}$ In this way, any fetus become its own control.

The biometric parameter of choice is the abdominal circumference (AC) as it has a good reproducibility and has also a good correlation with characteristics of the fetal growth.

In order to recognize any deviation from the expected curve of fetal growth, it is necessary to know the accurate GA. At the moment, the most accurate method for dating a pregnancy is the measurement of the crown-rump length in the first trimester. Its accuracy is plus or minus 4 days.

Plotting the observed value on growth charts, it is possible to build an expected trajectory of growth. Plotting on it, the values obtained by subsequent measurements, it is possible to detect any significant deviation. A restriction of the AC of $10 \%$ from the expected should be considered as suspicion of IUGR. An ultrasound scan for confirming this observation should not be done before 2 weeks. 


\section{ETIOLOGY OF IUGR}

After recognition of IUGR, it is necessary to identify the possible cause, the most frequent being placental obliterative vasculopathy leading to $\mathrm{CFH}$. In this condition, profound hemodynamic changes occur at the level of umbilical, fetal, and uteroplacental circulation.

In umbilical arteries, the peripheral resistance can be increased. According to the severity of the placental damage, the Doppler Velocity Wave Form can depict an increase in the pulsatility index (PI) or absence or reverse flow in diastole (ARED flow).

Today, Doppler study on umbilical arteries can be considered the best test of placental function.

\section{MANAGEMENT}

Currently, evidence that Doppler study improves perinatal outcome is only documented for the umbilical arteries.

Pulsatility index over the second standard deviation with the presence of diastolic flow is a warning sign of the probability of $\mathrm{CFH}$ and the necessity to evaluate the fetal well-being accurately.

Abnormal PI values with diastolic blood flow present alone does not suggest a prompt delivery. In this condition, vaginal delivery is achievable in 20 to $40 \%$ of the cases. ${ }^{4,5}$

The presence of ARED flow can suggest the opportunity of a prompt delivery, taking into consideration if end diastolic flow absent (EDFA) or reverse flow in diastole $(\mathrm{RF})$ is observed.

\section{RECOMMENDATIONS}

- Intrauterine growth restriction should be defined on the basis of serial measurements showing restricted growth - Level B.
- Abdominal circumference measurements are easy to perform and can be used for growth assessment Level B.

- Customized population-specific growth charts should be used when possible - Level B.

- The timely recognition of IUGR improves both management and outcomes. Screening for IUGR should be considered - Level A.

- When IUGR is suspected, the etiology should be explored - Level B.

- When the etiology is thought to be placental, Doppler study of the umbilical artery allows the identification or exclusion of significant CFH. Close monitoring of Doppler blood flow changes should guide the clinical management - Level A.

\section{REFERENCES}

1. Unterscheider J, Daly S, Geary MP, Kennelly MM, McAuliffe FM, O’Donoghue K, Hunter A, Morrison JJ, Burke G, Dicker P, et al. Definition and management of fetal growth restriction: a survey of contemporary attitudes. Eur J Obstet Gynecol Reprod Biol 2014 Mar;174:41-45.

2. Morales-Roselló J, Khalil A, Morlando M, Papageorghiou A, Bhide A, Thilaganathan B. Changes in fetal Doppler indices as a marker of failure to reach growth potential at term. Ultrasound Obstet Gynecol 2014 Mar;43(3):303-310.

3. Mandruzzato G, Antsaklis A, Botet F, Chervenak FA, Figueras F, Grunebaum A, Puerto B, Skupski D, Stanojevic M, WAPM. Intrauterine restriction (IUGR). J Perinat Med 2008; 36(4):277-281.

4. Li H, Gudmundsson S, Olofsson P. Prospect for vaginal delivery of growth restricted foetuses with abnormal umbilical artery blood flow. Acta Obstet Gynecol Scand 2003 Sep;82(9):828-833.

5. Mandruzzato G, Meir YJ, Natale R, Maso G. Antepartal assessment of IUGR fetuses. J Perinat Med 2001;29(3):222-229. 